\title{
Estrutura institucional na avaliação e monitoramento de políticas públicas: uma análise nos países do MERCOSUL
}

O presente artigo procura analisar a institucionalidade do sistema avaliação e monitoramento de políticas públicas (AMPP) nos países do MERCOSUL (Argentina, Brasil, Paraguai e Uruguai). Especificamente, interessa descrever a estrutura institucional vigente para detectar a possível convergência, heterogeneidade e limitações dos distintos arranjos institucionais de AMPP nos países analisados. Trata-se de uma pesquisa qualitativa, que utiliza o procedimento de análise de conteúdo para estimar um indicador de institucionalização. O estudo utiliza como fonte de dados as principais legislações nacionais, normativas, planos e diretrizes de política pública vigentes, assim como dados secundários e relatórios de organizações internacionais. Considera-se que, um sistema nacional de avaliação e monitoramento consolidado e unificado, pode impactar positivamente na performance das políticas públicas. Os principais resultados da análise mostram que existe heterogeneidade na institucionalidade de AMPP dos países, e quando comparados, o sistema nacional do Brasil apresenta o maior nível (alto), seguido da Argentina e Uruguai (médio) e por último Paraguai (baixo). Por fim, os países coincidem na ausência de uma política nacional de avaliação e monitoramento ampla e unificada, assim como na falta de uma agência nacional/federal que coordene o sistema nacional de AMPP.

\section{Institutional structure in the evaluation and monitoring of public policies: an analysis in MERCOSUR countries}

\begin{abstract}
This research aims to analyze the institutionalization of the public policy monitoring and evaluation (M\&E) system in the MERCOSUR countries (Argentina, Brazil, Paraguay and Uruguay). In particular, it is interesting to describe the current institutional structure to detect the possible convergence, heterogeneity and limitations of the different institutional M\&E arrangements in the countries analyzed. The research has a qualitative approach, which uses the content analysis procedure to estimate an institutionalization indicator. The main source of data was the national laws, regulations, plans and public policy guidelines in force, as well as secondary data from international organizations. It is considered that a consolidated and unified national M\&E system can have a positive impact on the performance of public policies. The main results of the analysis show that there is heterogeneity in the M\&E institutionalization, and when compared, Brazil's national system has the highest level (high), followed by Argentina and Uruguay (medium) and finally Paraguay (low). Finally, countries coincide in the absence of a broad and unified national M\&E policy, and also in the absence of a national/federal agency to coordinate the national M\&E system.
\end{abstract}

Keywords: Public policy; Evaluation; Monitoring; Institutions; Mercosur.

Topic: Gestão Pública

Reviewed anonymously in the process of blind peer.

Carlos Alberto Ramos Torres

Universidade Federal do Paraná, Brasil

http://lattes.cnpq.br/2260069288370308

http://orcid.org/0000-0001-8022-0131

carlosramosvp@gmail.com

Dalglish Fernando Vieira (iD

Universidade Federal do Paraná, Brasil

http://lattes.cnpq.br/0207610626530815

http://orcid.org/0000-0002-7981-0588

dalglish.fernando@gmail.com

Renata Jacobovski (iD

Universidade Federal do Paraná, Brasil

http://lattes.cnpq.br/9230837154809508

http://orcid.org/0000-0001-6028-5528

renatajacobovski@gmail.com
Received: 04/04/2021

Approved: 06/06/2021
6

DOI: 10.6008/CBPC2179-684X.2021.002.0019
Referencing this:

TORRES, C. A. R.; VIEIRA, D. F.; JACOBOVSKI, R.. Estrutura institucional na avaliação e monitoramento de políticas públicas: uma análise nos países do MERCOSUL. Revista Brasileira de Administração Científica, v.12, n.2, p.232-245, 2021. DOI: http://doi.org/10.6008/CBPC2179684X.2021.002.0019 


\section{INTRODUÇÃO}

O florescimento do campo de públicas como paradigma de pesquisa está historicamente relacionado com o aumento e diversificação da ação Estatal. A origem histórica da avaliação de políticas públicas pode remontar a sociedades antigas, como a China imperial ou a Inglaterra do século XVIII (FITZPATRICK et al., 2011). No entanto, foi no contexto do século XX (com forte relação à expansão estatal estadunidense) que houve uma notável aceleração no episteme das políticas públicas bem como no processo de avaliação como prática, profissão e disciplina técnica do governo.

A América Latina e o Caribe (ALC) não ficaram à margem desses processos de expansão da atividade pública e seus desdobramentos no âmbito da avaliação e monitoramento de políticas públicas (AMPP). Contudo, o surgimento expressivo das instituições orientadas ao aprimoramento de políticas públicas, tiveram seu boom na redemocratização e emergência de paradigmas como a Nova Gestão Pública (NGP) desde a década de 90's (LÁZARO, 2015). A União Europeia (UE) tem desenvolvido ao longo de duas décadas um sistema coordenado e institucionalizado de AMPP que tem influenciado positivamente na maioria dos seus membros (ZAPICO, 2010). Diferentemente, no Mercado Comum do Sul (MERCOSUL), uma organização internacional para a integração regional não pautada pela supranacionalidade nem pelo direito comunitário, seus membros (Argentina, Brasil, Paraguai e Uruguai), em se tratando da AMPP, apresentam níveis institucionais bastante heterogêneos.

Considerando a escassez de estudos comparados que analisem, especificamente, o caso das instituições de monitoramento e avaliação nos países do MERCOSUL, a presente pesquisa almeja responder:(i) - Qual a estrutura institucional vigente nos sistemas de AMPP dos países do MERCOSUL? (ii) Existe alguma convergência institucional e padrões comuns entre os sistemas e mecanismos de AMPP desses países? (iii) - quais são os principais desafios institucionais no que diz respeito a sistemas e mecanismos regionais de AMPP no contexto do MERCOSUL?.

\section{METODOLOGIA}

A presente pesquisa é de caráter qualitativa e descritiva, pautada sobretudo pela revisão bibliográfica. O principal procedimento adotado consistiu na análise de conteúdo. Para realização da análise, foram consideradas como base de dados as principais legislações nacionais, normativas, planos e diretrizes vigentes nos países do MERCOSUL no âmbito da AMPP.

Mediante uma tabela analítica, almeja-se descrever o grau de institucionalização dos sistemas nacionais de AMPP nesses países, utilizando quatro dimensões analíticas e indicadores, extraídos, principalmente, a partir dos trabalhos de Carrasco (2015) e Lázaro (2015). Na tabela analítica identifica-se a presença (peso 1) ou ausência (peso 0) de indicadores de institucionalização conforme as fontes descritas anteriormente. Para a tabulação dos resultados, faz-se a somatória do peso dos indicadores e segue-se a escala de institucionalização proposta pelo Carrasco (2015): 1-4 baixo; 5-7 médio; e, 8-12 alto.

\section{Conceitos, definições e o panorama da avaliação e monitoramento na ALC}


Não existe uma única definição sobre a prática da avaliação e existem diferentes critérios para sua conceituação. Genericamente, a avaliação pode ser entendida como o processo para determinar em que medida as metas e os objetivos de um programa ou projeto foram alcançados (TYLER, 1950). Normalmente, a avaliação é considerada a fase de análise de políticas orientada para pesquisa, operacionalizada mediante testes, no intuito de achar respostas causais que podem ser ex-post e, as vezes, ex-ante (GEVA-MAY et al., 1999). Por sua vez, compreende-se por monitoramento uma função contínua que usa a coleta sistemática de dados sobre indicadores específicos para fornecer aos gestores e principais interessados em uma intervenção as indicações sobre o progresso e a realização dos objetivos, bem como quanto ao emprego dos recursos alocados (OCDE, 2002).

Grosso modo, um sistema nacional de avaliação e monitoramento de políticas públicas pode ser definido como o resultado da aplicação de princípios, processos, procedimentos e práticas operativas e administrativas, destinadas ao desenvolvimento de um conjunto de ferramentas integradas pelas quais governos, cidadãos, organizações relacionadas com a vida pública, organizações internacionais, e qualquer ator relacionado com o processo político, podem fazer seguimento de resultados da implementação de políticas e programas públicos no decorrer do tempo (BID, 2016).

Uma política nacional de avaliação pode ser definida como uma diretriz jurídica que serve como base para o processo de avaliação de políticas e programas no âmbito de diversas agências ou unidades de governo, de forma coordenada e sustentada no tempo (AQUILINO, 2015). Neste sentido, considera-se institucionalização todas as ações da esfera pública que incidem na criação e constituição de um sistema de AMPP, sempre e quando os resultados do sistema sejam valorados e utilizados pelas esferas de governo (MACKAY, 2006; CARRASCO, 2015).

Assim, o propósito das instituições de AMPP deve estar orientado à provisão de informações úteis e confiáveis sobre resultados, impactos e falhas na implementação de políticas, tanto para os tomadores de decisão, quanto para cidadãos e sociedade civil envolvida no processo político. Como assinalam Queiroz et al. (2020), os resultados obtidos pelas políticas e programas públicos dependem, muitas vezes, das formas e variedades institucionais que permeiam seu processo de desenvolvimento.

A literatura apresenta uma série de estudos comparativos entre países latino-americanos que abordam, direta ou indiretamente, a hipótese de que a presença de sistemas nacionais de AMPP pode melhorar a performance das políticas públicas. O trabalho pioneiro de Cunill et al. (2008) analisou 12 países da ALC, enfatizando a heterogeneidade da institucionalidade dos sistemas. Da mesma forma o BID (2011), incorporando quase todos os países da ALC, realizou um estudo da capacidade de gestão para resultados. Analogamente, Feinstein (2015) apresenta um panorama geral da AMPP na ALC, enfatizando a necessidade de superar pragmaticamente o fundamentalismo metodológico na prática da avaliação e a separação de conceitos entre monitoramento e avaliação. Normalmente, os estudos de caso relacionados a AMPP na ALC são realizados em nível nacional (CARRASCO, 2015; LÁZARO, 2015; YARAHUÁN et al., 2015; HAEFNER et al., 2019; NEIROTTI, 2020). 
Especificamente, nos países do MERCOSUL, a literatura consultada aponta que os casos da Argentina, Paraguai e Uruguai são sistemas de AMPP em redesenho constante e no caso do Brasil, o sistema é mais 'estável' e coeso. A Figura 1 mostra que nos países da ALC, quando se evidencia avanços e aprimoramentos no sistema de AMPP também há melhoras na eficácia dos governos. Isso não quer dizer que haja uma relação causal entre ambas variáveis, mas apenas uma tendência regional constatada empiricamente na maioria dos países da amostra. Para tal se construiu uma correlação com dados do Banco Mundial (BM) e do BID.

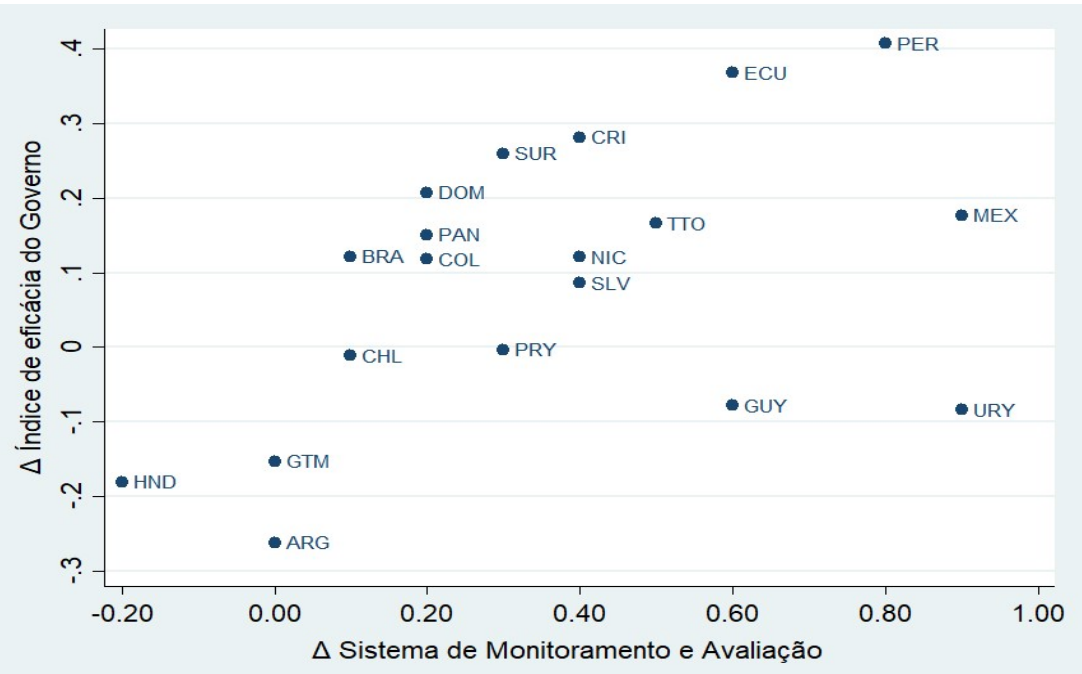

Figura 1: Taxa de variação do Índice de Eficácia do Governo e do Índice de Sistemas de Monitoramento e Avaliação, vários países da ALC (2014).

É importante observar que dos países do MERCOSUL, o Uruguai apresentou melhoras no seu sistema de AMPP (apesar de não ter melhoras equivalentes no indicador de eficácia do governo). No caso brasileiro, se observa a tendência de melhora em ambos indicadores; já no caso argentino, houve pioras na eficácia do governo, mantendo constante o desempenho do seu sistema de AMPP. Por último, no caso paraguaio, verificou-se uma melhora no sistema de AMPP, mantendo constante seu índice de eficácia governamental.

Por outro lado, conforme dados do Índice de Gestão por Resultados (IGR) 2010 do BID, existe assimetria no desempenho do setor público entre os países do MERCOSUL. Considerando, especificamente, a dimensão de monitoramento e avaliação, é notável a diferença positiva do Brasil se comparado com os outros países. A Tabela 1 apresenta com detalhe os dados acima mencionados:

Tabela 1: Índice de Gestão por Resultados (IGR) ano 2010.

\begin{tabular}{|c|c|c|c|c|c|c|c|c|c|}
\hline Pais & Planejamento & Orçamento & $\begin{array}{l}\text { Gestão financeira } \\
\text { auditoria }\end{array}$ & e & $\begin{array}{l}\text { Gestão de programas } \\
\text { projetos }\end{array}$ & e & $\begin{array}{l}\text { Monitoramento } \\
\text { avaliação }\end{array}$ & e & IGR \\
\hline Argentina & 2,2 & 2,1 & 3,3 & & 1,5 & & 1,2 & & 2,1 \\
\hline Brasil & 4,2 & 3,8 & 4 & & 2,9 & & 3,9 & & 3,8 \\
\hline Paraguai & 1,7 & 1,1 & 2,3 & & 1,1 & & 0,4 & & 1,3 \\
\hline Uruguai & 2,1 & 1,8 & 2,5 & & 1,4 & & 1,3 & & 1,8 \\
\hline ALC & 2,3 & 1,4 & 2,5 & & 1,9 & & 1,6 & & 1,9 \\
\hline
\end{tabular}

Fonte: BID (2011).

O informe do IGR também aponta para a falta de complementariedade e coordenação institucional nos sistemas AMPP da Argentina e Uruguai; além disso, detecta, no caso paraguaio, que tais mecanismos quase não existem ou se encontram em estágios iniciais. 


\section{DISCUSSÃO TEÓRICA}

\section{Análise da institucionalização de AMPP nos países do MERCOSUL}

Diante do já exposto, a seguir se apresentará uma breve análise qualitativa das principais características do arranjo institucional vigente em matéria de AMPP nos países do MERCOSUL. No Quadro 1, é possível inferir um panorama geral da institucionalidade de AMPP nos países analisados. É possível verificar, a priori, a ausência de harmonia e unificação dos sistemas de avaliação dos países, isto pode ser decorrente da falta de uma política nacional de AMPP abrangente e unificada nos diversos níveis do setor público.

Quadro 1: Panorama institucional de AMPP nos países do MERCOSUL.

\begin{tabular}{|c|c|c|c|c|}
\hline País & $\begin{array}{l}\text { Nome do sistema de } \\
\text { AMPP }\end{array}$ & $\begin{array}{|ll|}\text { Organismo } & (\mathrm{s}) \\
\text { responsável }\end{array}$ & \begin{tabular}{|l|} 
Instrumento \\
genérico \\
planejamento \\
\end{tabular} & $\begin{array}{l}\text { Marco jurídico: Leis, normativas e } \\
\text { diretrizes }\end{array}$ \\
\hline $\begin{array}{l}\text { Argentin } \\
\text { a }\end{array}$ & $\begin{array}{l}\text { Não possui um sistema } \\
\text { nacional unificado. }\end{array}$ & $\begin{array}{l}\text { Jefatura de Gabinete de } \\
\text { Ministros (JGM) }\end{array}$ & $\begin{array}{|lr|}\text { Cuenta de Inversión } \\
\text { anual/Planes } \\
\text { Nacionales da } \\
\text { Secretaria de } \\
\text { Públicas }\end{array}$ & $\begin{array}{l}\text { Art. } 100 \text { da Constituição/Lei } 24.156 \text { de } \\
1992 \text { e Lei } 25.152 \text { de 1999/Resolução } \\
\text { JGM 416/2013/Plan de Modernização de } \\
2016\end{array}$ \\
\hline Brasil & $\begin{array}{l}\text { Não possui um sistema } \\
\text { nacional unificado. }\end{array}$ & $\begin{array}{l}\text { Conselho de } \\
\text { Monitoramento e } \\
\text { Avaliação de Políticas } \\
\text { Públicas } \\
\text { (CMAP)/Secretaria da } \\
\text { Fazenda Pública } \\
\text { (SEFAZ/ME) }\end{array}$ & $\begin{array}{|ll|}\text { Plano } & \text { Plurianual } \\
\text { (PPA), Lei de } \\
\text { Diretrizes } \\
\text { Orçamentárias (LDO), } \\
\text { Ações de Órgãos de } \\
\text { Controle } & \text { Interno e e } \\
\text { Externo (CGU e TCU) }\end{array}$ & $\begin{array}{l}\text { Art. } 165 \text { da CRFB/88/Lei no } 13.971 / 2019 \\
\text { - PPA (2020-2023)/Lei no } 14.116 / 2020 \text { - } \\
\text { LDO (2021)/Decreto no } 9.834 / 2019 \text { - } \\
\text { CMAP. }\end{array}$ \\
\hline Paraguai & $\begin{array}{l}\text { Não possui um sistema } \\
\text { nacional unificado. }\end{array}$ & $\begin{array}{lr}\text { Secretaria Técnica } & \text { de } \\
\text { Planificación } & y \\
\text { Desarrollo (STPD). } & \\
\end{array}$ & $\begin{array}{l}\text { Plan de Desarrollo } \\
2030\end{array}$ & $\begin{array}{l}\text { Lei no 841, de } 14 \text { de setembro de } \\
\text { 1962/Decreto } \\
\text { N6258/2019/Decreto 1145/2019. }\end{array}$ \\
\hline Uruguai & $\begin{array}{l}\text { Não possui um sistema } \\
\text { nacional unificado. }\end{array}$ & $\begin{array}{l}\text { Área de Gestión y } \\
\text { Evaluación del Estado } \\
\text { (AGEV)/Dirección } \\
\text { Nacional de Evaluación y } \\
\text { Monitoreo (DINEM) }\end{array}$ & $\begin{array}{l}\text { Ley de Presupuesto } \\
\text { Nacional/Registro } \\
\text { Nacional de } \\
\text { Evaluaciones }\end{array}$ & $\begin{array}{l}\text { Ley No } 16.736 \text { de 1996/ ley } 17.866 \text { de } \\
\text { 2005/Art. 58, Ley } N^{\circ} 17.930 \text { de } 19 \text { de } \\
\text { diciembre de 2005/ Ley } 18.996 \text { del } 7 \text { de } \\
\text { noviembre de 2012/ Ley de urgente } \\
\text { consideración (LUC) 2020, artículo } 305 \text {. }\end{array}$ \\
\hline
\end{tabular}

Conquanto o exposto até o momento, no intuito de melhor compreender o sistema de AMPP instalado em cada país, em seguida, apresenta-se uma análise descritiva de cada sistema de AMPP nos países membros do MERCOSUL, no intuito de descrever a institucionalidade vigente.

\section{Institucionalidade da AMPP na Argentina}

Em linhas gerais, o quadro institucional de AMPP na Argentina mostra-se fragmentado e desarticulado, carente de uma arquitetura normativa capaz de direcionar de forma organizada a função de avaliação no Estado (AQUILINO et al., 2017; GUERSANI, 2019). Nesse sentido, até o final de 2019 não havia como se falar em um sistema ou órgão nacional de AMPP, muito embora as diversas tentativas falidas ${ }^{1}$ para sua criação. No entanto, em março de 2020, o recém-eleito Presidente Alberto Fernandez anunciou um projeto de Lei para a criação de uma Agencia Nacional de Evaluación de Políticas Públicas (ANEPP).

A nível constitucional, desde 1995 atribuíram-se algumas responsabilidades relacionadas ao processo de monitoramento e avaliação à Jefatura de Gabinete de Ministros (JGM). Contudo, como bem assinalado

\footnotetext{
${ }_{1}^{1}$ Projetos de lei apresentados: 3046-D-2010, 2268-D-2011, 2422-S2015 y 0751-S-2014 y S-882/17 para a criação da Agencia, sem sucesso. 
por Aquilino et al. (2017), em mais de duas décadas de atuação da JGM a cultura da avaliação ainda não é percebida, e tampouco parece haver interesse para a consolidação de um sistema nacional de AMPP. A Argentina também conta com o Sistema de Información, Monitoreo y Evaluación de Programas Sociales (SIEMPRO), criada em 1995 no intuito de fortalecer a política social (CASTRO, 2015).

Entretanto, além da instabilidade e constantes mudanças administrativas fruto dos ciclos políticos argentinos, tal órgão tem desenvolvido um papel residual na AMPP, bastante limitado à interação com a JGM (AQUILINO et al., 2017; CASTRO, 2015). Na atual gestão do Fernández, o SIEMPRO parece ter mais participação, com uma agenda de atuação que interage com diversas esferas da administração pública argentina. Uma consulta no site da entidade ${ }^{2}$, mostra o planejamento de pelo menos 72 avaliações, nas mais diversas áreas, programadas desde início de 2020 até finais de 2021.

No que diz respeito ao orçamento, a Argentina mediante a Oficina Nacional de Presupuesto (ONP) tem desenvolvido um sistema de monitoramento trimestral dos programas orçamentários (AQUILINO, 2015). Tal sistema da ONP é dependente do Ministério da Fazenda argentino, e se rege mediante a Lei 24.156 de Administración Financiera y Sistemas de Control, promulgada em outubro de 1992. Por último, vale a pena mencionar o Programa de Evaluación de Calidad del Gasto, uma iniciativa do Ministério da Economia que, em linhas gerais, visa fazer diretrizes e estimações de custo-benefício para melhorar a produção de bens e serviços públicos.

A maioria destes aparelhos jurídicos-institucionais para AMPP na Argentina, estão defasados em comparação a outros sistemas latino-americanos mais robustos e consolidados como o da Colômbia, Chile

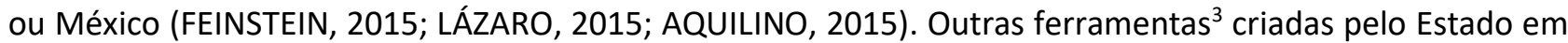
matéria de avaliação foram de caráter circunstancial e instrumental, sem projeção de longo prazo (por isso foram se extinguindo nos ciclos políticos ou estão quase inutilizadas atualmente).

Junto com o anúncio de criação da ANEPP em 2020, a Argentina vem sendo influenciada por organizações internacionais como a Open Government Partnership (OGP) e o Centro para el Aprendizaje en Evaluación y Resultados (CLEAR). Uma série de fóruns e conferências foram promovidas recentemente pelas referidas instituições e outros setores como universidades, a sociedade civil organizada, dentre outros. Além disso, o surgimento de novos programas de pós-graduação em avaliação de políticas públicas, parecem configurar o cenário para o florescimento de maiores níveis de maturidade no âmbito institucional e prático da AMPP na Argentina 4 .

\section{Institucionalidade da AMPP no Brasil}

Em relação ao Brasil, têm-se que a partir da redefinição das funções e atribuições do aparato administrativo estatal, ocorrida sobretudo nas duas décadas passadas, ocasião na qual instrumentos da

\footnotetext{
${ }^{2}$ https://www.argentina.gob.ar/politicassociales/siempro/publicaciones/evaluaciones-programas-sociales-estado-nacional-2020

${ }^{3} \mathrm{Em}$ 2010, o Sistema de Informação para Gestão (SIG) e o Programa de Suporte à Implementação da Gestão para Resultados. Em 2013, a Resolução JGM 416/2013: Programa de Avaliação de Políticas Públicas. Já em 2016, o Decreto 434/2016 do Plano de Modernização do Estado, mediante um eixo de Gestão por Resultados e Avaliação de Políticas Públicas; e no mesmo ano, a Lei de Acesso à Informação Pública.

${ }^{4}$ Uma discussão mais detalhada sobre o cenário recente pode ser encontrada em Neirotti (2020).
} 
administração privada passaram a ser implementados nas organizações do setor público, ferramentas de AMPP passaram a ser consideradas fundamentais na gestão de políticas públicas na qual a medição dos resultados e indicadores de desempenho são pressupostos essenciais (NOGUEIRA et al., 2009).

Assim, muito embora boa parte da literatura defenda a inserção de mecanismos de AMPP apenas formalmente, é possível afirmar que esse processo de institucionalização está bem avançado na administração pública brasileira, principalmente, em função da criação de sistemas avaliativos sofisticados e integrantes do ciclo de políticas públicas. Nesse sentido, afirma-se que a análise e o monitoramento de programas consistem na verificação da adequação do desenho do programa à sua finalidade e aos objetivos pretendidos, da correlação entre os serviços prestados e as metas definidas no planejamento estratégico, dos efeitos mensuráveis e, por fim, da lógica de eficiência subjacente, em outras palavras, verificar se os benefícios da política justificam seu custo (NOGUEIRA et al., 2009).

No Brasil das últimas décadas, é possível afirmar que o processo de AMPP, necessariamente passa pela elaboração e utilização de métodos e ferramentas empregados no planejamento estratégico, até então pouco (ou nada) utilizados pelo Poder Público. Dentre os principais instrumentos utilizados para esse tipo de verificação, destaca-se o Plano Plurianual - PPA, cuja elaboração está prevista no artigo 165, inciso I, da Constituição Federal da República Federativa do Brasil (CRFB/88) (BRASIL, 1988) e, necessariamente, deverá observar as diretrizes, objetivos e metas da administração pública, bem como os planos e programas nacionais, regionais e setoriais previstos no próprio texto constitucional, os quais vigerão pelo prazo de quatro anos.

Nos termos do artigo 4으, da Lei no 13.971, de 27 de dezembro de 2019 (BRASIL, 2019b), que instituiu o PPA (2020-2023), O PPA 2020-2023 reflete políticas públicas, orienta atuação governamental e define diretrizes, objetivos, metas e programas. Além disso, dispõe que a cada programa finalístico será associada uma unidade responsável, um objetivo e uma meta, ou seja, em se tratando de planos e políticas do Governo Federal, têm-se, ainda, que as ações e programas são agrupados e gerenciados na maioria das vezes por unidades finalísticas vinculadas a Ministérios e/ou Poderes, cabendo ao Ministério da Economia a consolidação dessas informações.

Funcionando como uma espécie de instrumento de execução do Plano Plurianual, anualmente o Executivo encaminha ao Congresso Nacional para ampla discussão o Projeto de Lei de Diretrizes Orçamentárias - LDO, cujo objetivo principal é estabelecer as diretrizes para a confecção do Orçamento público, o qual também se consubstancia em uma lei, a Lei Orçamentária Anual - LOA. Devidamente publicizadas, as referidas leis possibilitam a qualquer cidadão o acompanhamento das contas públicas, bem como de reclamar eventuais desmandos às autoridades competentes.

Além dos referidos instrumentos, e dos avanços na área de políticas públicas, é possível afirmar que nas últimas décadas, em especial na última, o Governo Federal evoluiu substancialmente sobretudo em se tratando de controle de gastos públicos e, consequentemente, na avaliação e no monitoramento das políticas públicas. Exemplo desse avanço é o Conselho de Monitoramento e Avaliação de Políticas Públicas (CMAP), instituído pelo Decreto no 9.834/2019 (BRASIL, 2019a), bem como a incorporação do Comitê de 
Monitoramento e Avaliação dos Subsídios da União (CMAS), que havia sido instituído pelo Decreto no 9.588, de 27 de novembro de 2018 (BRASIL, 2018), pelo primeiro ${ }^{5}$, embora o pouco tempo de criação dessas instâncias.

O CMAP desempenha suas funções de avaliação por meio de comitês, como é o caso do CMAS, mencionado acima, bem como do Comitê de Monitoramento e Avaliação de Gastos Diretos (CMAG). Além disso, o Conselho também monitora a implementação das alterações em políticas públicas que recomendar, a partir da avaliação realizada em políticas previamente selecionadas, por critérios determinados e a partir de programas finalísticos do PPA.

Desde 2019, quando da sua instituição, o CMAP é composto por órgãos da administração direta, como é o caso do Ministério da Economia, da Casa Civil e da Controladoria Geral da União (CGU), sob a coordenação do primeiro, reiterando o protagonismo do Ministério da Economia nesse processo. Além disso, ressalta-se o apoio de outros órgãos, como é o caso da Escola Nacional de Administração Pública (ENAP) e do Instituto de Pesquisa Econômica Aplicada (IPEA), bem como do Banco Interamericano de Desenvolvimento $(\mathrm{BID})^{6}$.

Inobstante todo o exposto, há que se ressaltar, ainda, o trabalho desenvolvido pela CGU, por intermédio da Secretaria Federal de Controle Interno $(\mathrm{SFC})^{7}$, a quem cabe, dentre outras funções, avaliar a execução de programas de governo, bem como sua atividade por intermédio do Observatório da Despesa Pública $(\mathrm{ODP})^{8}$, cujo objetivo principal é contribuir para o aprimoramento do controle interno, e, ainda, ações de fomento ao Controle Social, ou seja, conscientizar os cidadãos da importância participação da sociedade no processo de fiscalização da efetividade e eficiência das ações governamentais ${ }^{9}$. Por fim, não se pode olvidar os papéis fundamentais desempenhados pelas Organizações não Governamentais (ONGs), sociedade civil organizada e universidades, seja na fiscalização, no aperfeiçoamento ou na propositura de novas políticas públicas.

\section{Institucionalidade da AMPP no Paraguai}

No caso do Paraguai, foi instituída como dependente da Presidência da República, a Secretaria Técnica de Planificación y Desarrollo (STPD). Trata-se de um órgão criado pela Lei no 841, de 14 de setembro de 1962, mas que no decorrer do tempo passou por importantes transformações institucionais, sendo as mais recentes datadas de 2004 (decreto número 4070/04) e 2015 (resolução 52/2015). Atualmente, o órgão conta com uma estrutura institucional que incorpora uma Dirección General del Análisis de Políticas Públicas (DGAPP), porém, encontra-se longe de contar com uma política nacional de avaliação.

A STPD é encarregada de formular e coordenar o Plano Nacional de Desenvolvimento, diretriz máxima de política pública nacional no Paraguai. Conjuntamente com o Ministério da Fazenda do Paraguai,

\footnotetext{
${ }^{5}$ https://www.gov.br/economia/pt-br/acesso-a-informacao/participacao-social/conselhos-e-orgaos-colegiados/cmap/institucional/comite-demonitoramento-e-avaliacao-de-subsidios-da-uniao-cmas

${ }^{6}$ https://www.gov.br/economia/pt-br/acesso-a-informacao/participacao-social/conselhos-e-orgaos-colegiados/cmap/politicas

${ }^{7}$ https://www.gov.br/cgu/pt-br/assuntos/auditoria-e-fiscalizacao

${ }^{8}$ https://www.gov.br/cgu/pt-br/assuntos/informacoes-estrategicas/observatorio-da-despesa-publica

${ }^{9}$ https://www.gov.br/cgu/pt-br/assuntos/controle-social
} 
a STP elabora um informe anual sobre gestões e ações prioritárias, além de um informe sobre saúde fiscal e contas nacionais em relação aos programas de investimento e criação de bens públicos (MÁTTAR et al., 2017). Toda a atividade da STPD está orientada a seguir a metodologia de planejamento por resultados, com diretrizes operacionais e formatos ${ }^{10}$ para avaliação regidos segundo a Lei N6258/2019 e o Decreto $1145 / 2019$.

No marco do Plano Nacional de Desenvolvimento vigente no Paraguai, propõe-se a criação do Consejo Nacional de Evaluación de la Gestión Pública que auxiliará ao DGAPP nas tarefas de avaliação de políticas públicas executadas no marco do plano. A nível constitucional, o Art. 238 prevê que a STPD junto com o Gabinete Civil é responsável pela prestação de contas entregue ao Congresso pelo Presidente, constituindo, indiretamente, uma forma expressa de avaliação, porém longe de ser uma diretriz clara constitucional. Os decretos citados anteriormente, como o 4.070/04 de 2004, viabilizaram a emergência de algumas ferramentas como o 'Tablero de control', destinada a monitorar o Plano Nacional de Redução da pobreza e determinar os níveis de eficácia do gasto.

Outra das iniciativas isoladas, executada pela STPD, é uma ferramenta chamada $\mathrm{HECl}^{11}$ para avaliar a qualidade dos indicadores, metas e fontes de informação. Trata-se de uma planilha que incorpora um questionário com perguntas, não ficando muito claro qual o destinatário das informações produzidas. Uma consulta no site da STPD mostra o link ${ }^{12}$ de acesso ao formulário e aparentemente está aberto para qualquer um que pertença a uma instituição no Paraguai. A avaliação pode ser inserida na sociedade mediante o fomento de ensino e pesquisa no campo de públicas, contudo, observa-se que há escassez de pesquisas que abordem a questão da AMPP no Paraguai, assim como de programas de pós-graduação da área (ZAMORANO, 2012; LAFUENTE, 2009; MASI et al., 2016).

Percebe-se assim que não existe um sistema nacional de AMPP articulado e organizado, inobstante a existência de uma instituição criada por lei para cumprir tal função. A priori, a institucionalidade de AMPP paraguaia é a menos desenvolvida dos países analisados. Isto não quer dizer que o Paraguai não realiza avaliação e monitoramento de políticas, mas que o país não conta com uma diretriz nacional abrangente para os diversos níveis da administração pública.

\section{Institucionalidade do AMPP no Uruguai}

O regime político do Uruguai apresenta certa estabilidade democrática ${ }^{13}$, mediante um sistema forte de partidos e um setor público com saudáveis indicadores e baixos níveis de percepção da corrupção. Na "virada à esquerda" em 2005, com a chegada do projeto político da Frente Ampla (FA), a modernização do setor público entrou nas prioridades da Agenda pública, e em matéria de avaliação criou-se a Dirección Nacional de Evaluación y Monitoreo (DINEM), vinculada ao Ministério de Desenvolvimento Social e a Área de Gestión y Evaluación del Estado (AGEV) (FONT, 2015). Da mesma forma, foi criada a Unidad de Asesoramiento

\footnotetext{
${ }^{10}$ Os formatos estão disponíveis no site da STP https://www.stp.gov.py/v1/instrumentos-spr/

${ }^{11}$ Disponível em: https://docs.google.com/spreadsheets/d/1EekHUDtqfcv_MC5WWuw LH37Bq3oiiQKx7iNmCj8bt0/edit\#gid=1563520212

${ }^{12}$ Disponível em: https://docs.google.com/forms/d/e/1FAIpQLSfY M3nUH16Jz80In4XWpufy-Tw0mA4f OS5YMF F4ZpNDERg/viewform?c=0\&w=1

${ }^{13}$ A única democracia latino-americana "completa" em 2017, no índice de democracias publicado pelo The Economist.
} 
y Monitoreo de Políticas (UAMP) em 2005, dependente da Presidência da República.

No entanto, o Uruguai não conta com um sistema nacional de AMPP que seja referência na região. Umas das possíveis causas é a falta de incentivos para ampliar o alcance da AMPP, consequência dos bons índices de transparência e confiança cidadã no Estado (FREIGEDO et al., 2017). No país existem sistemas de informação governamental sobre a situação socioeconómica, mas segundo o BID (2010) tais informações não são utilizadas sistematicamente para avaliar e monitorar o avanço de metas e objetivos do governo ${ }^{14}$.

Além disso, não há na configuração jurídico-institucional uma lei que estabeleça a necessidade de avaliar programas e políticas ${ }^{15}$, identifica-se, também, a falta de metodologias e instrumentos para tal fim. $O$ Plano Nacional de Desenvolvimento de Capacidades para o Monitoramento e Avaliação (DCME) ${ }^{16}$, foi projetado em 2017 pela OPP (Oficina de Planeamiento y Presupuesto) da Presidência para fortalecer as práticas de AMPP no Uruguai. O DCME abarca uma série de objetivos e propostas práticas sugeridas pelos experts, incluindo a sugestão de modelos uniformes de avaliação mediante manuais de padronização, porém não se evidenciou no site da AGEV o status do plano e quais objetivos e ações foram de fato executadas.

No projeto político do recém eleito Presidente Lacalle Pou, existe uma proposta de criação da Agencia de Monitoreo y Evaluación de Políticas Públicas (AMEPP) em substituição da UAMP, isto segundo o Artigo 305 da Ley de Urgente Consideración (LUC) ${ }^{17}$. Entre alguns detalhes que apresenta o projeto de criação da AMEPP, encontra-se um Conselho Executivo presidido pelo Secretário da Presidência da República e um Diretor e subdiretor executivos nomeados pelo Presidente como cargos de confiança. A princípio, tal estrutura organizacional compromete a possibilidade de criar um sistema nacional independente dos ciclos políticos e com capacidade de organizar a política nacional de AMPP nos diversos níveis da administração pública.

Não obstante, durante o governo do FA foram criados órgãos com altíssima eficiência técnica e certa independência política, como por exemplo a Agência do Governo Eletrônico e Sociedade da Informação (AGESIC) mundialmente reconhecida pelos seus resultados. Assim, pode-se dizer que o Uruguai já conta com certo aprendizado institucional sobre este tipo de arranjo técnico-político. Outrossim, pode-se inferir da institucionalidade projetada na AMEPP que este órgão seja mais forte em monitoramento, e apresente mais 'limitações' na parte da avaliação (principalmente pela vinculação política e a questão temporal), ficando a dúvida se a AMEPP poderá avaliar políticas do passado ou ficará restrita apenas a servir ao governo em turno.

Diante de todo o exposto, na Tabela 2 a continuação, é possível verificar com mais clareza o nível de institucionalidade dos sistemas de AMPP nos países do MERCOSUL, incorporando os resultados tabelados da análise de conteúdo conforme às categorias analíticas avaliando os indicadores conforme os pesos indicados na seção metodológica.

\footnotetext{
14 Como exemplo de iniciativas, o Uruguai conta com um banco nacional de avaliações disponíveis em: https://transparenciapresupuestaria.opp.gub.uy/inicio/registro-nacional-de-evaluaciones

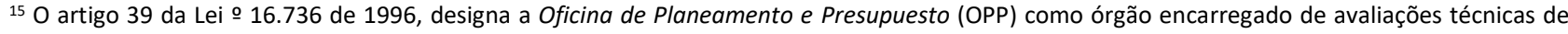
programas e políticas no marco do orçamento nacional.

${ }^{16}$ https://transparenciapresupuestaria.opp.gub.uy/sites/default/files/bloque-documentos/Plan\%20DCME resumen.pdf

${ }_{17}$ Trata-se de um pacote de medidas aprovadas com caráter extraordinário que servem como a próxima rota para diretrizes nacionais de política. 
Tabela 2: Análise institucional dos sistemas de AMPP nos países do MERCOSUL.

Dimensões de análise

Peso 0 (não)/1 (sim)

\begin{tabular}{|c|c|c|c|c|c|c|c|}
\hline Dimensão & & Critério & Indicadores & Argentina & Brasil & Paraguai & Uruguai \\
\hline \multirow{3}{*}{$\begin{array}{l}\text { 1- Presença } \\
\text { avaliação } \\
\text { constituição }\end{array}$} & \multirow{3}{*}{$\begin{array}{l}\text { de } \\
\text { na }\end{array}$} & \multirow{3}{*}{$\begin{array}{l}\text { Indica a existência de um } \\
\text { quadro de obrigações } \\
\text { constitucionais que apoia a } \\
\text { institucionalização } r \text { dos } \\
\text { sistemas nacionais de } \\
\text { avaliação. }\end{array}$} & $\begin{array}{l}\text { Há menção na Constituição } \\
\text { da avaliação de políticas } \\
\text { públicas. }\end{array}$ & 1 & 1 & 0 & 0 \\
\hline & & & $\begin{array}{l}\text { Existem artigos específicos } \\
\text { que regulamentam o } \\
\text { processo de avaliação. }\end{array}$ & 0 & 0 & 1 & 1 \\
\hline & & & $\begin{array}{l}\text { Existem artigos que } \\
\text { atribuem institucionalmente } \\
\text { a responsabilidade pela } \\
\text { avaliação a uma agência } \\
\text { governamental. }\end{array}$ & 0 & 0 & 1 & 1 \\
\hline \multirow{4}{*}{$\begin{array}{l}\text { 2- Presença } \\
\text { mecanismos } \\
\text { avaliação no } \\
\text { nacional } \\
\text { desenvolvimento }\end{array}$} & \multirow{3}{*}{$\begin{array}{r}\text { de } \\
\text { de } \\
\text { plano } \\
\text { de }\end{array}$} & \multirow{3}{*}{$\begin{array}{l}\text { Indica se os planos nacionais } \\
\text { incorporam e valorizam } \\
\text { mecanismos de avaliação em } \\
\text { seu desenho e monitoramento. }\end{array}$} & $\begin{array}{l}\text { Define e estabelece os } \\
\text { escopos da avaliação para a } \\
\text { melhoria dos resultados da } \\
\text { política. }\end{array}$ & 1 & 1 & 0 & 1 \\
\hline & & & $\begin{array}{l}\text { Existem indicadores de } \\
\text { avaliação para cada meta do } \\
\text { plano. }\end{array}$ & 0 & 1 & 0 & 0 \\
\hline & & & $\begin{array}{l}\text { Há clareza no acesso às } \\
\text { informações das avaliações } \\
\text { de conformidade com o } \\
\text { plano de política nacional. }\end{array}$ & 0 & 1 & 0 & 0 \\
\hline & & & $\begin{array}{l}\text { Existe um quadro jurídico } \\
\text { geral e harmonizado que } \\
\text { apoia e define os processos } \\
\text { de avaliação. }\end{array}$ & 0 & 1 & 0 & 0 \\
\hline
\end{tabular}
geral sobre validade e respaldo jurídico órgãos governamentais.

suportam os aspectos avaliação

\begin{tabular}{|c|c|c|c|c|}
\hline de avaliação & 1 & 1 & 1 & 1 \\
\hline $\begin{array}{l}\text { Existe legislação específica } \\
\text { para a criação e manutenção } \\
\text { de um sistema nacional de } \\
\text { avaliação. }\end{array}$ & 1 & 1 & 0 & 1 \\
\hline $\begin{array}{l}\text { Existe um sistema de } \\
\text { avaliação nacional. }\end{array}$ & 0 & 1 & 0 & 0 \\
\hline $\begin{array}{l}\text { Existem instituições } \\
\text { específicas responsáveis } \\
\text { pelos processos de } \\
\text { avaliação. }\end{array}$ & 1 & 1 & 1 & 1 \\
\hline $\begin{array}{l}\text { Há um uso racional e público } \\
\text { das informações geradas } \\
\text { pelas instituições } \\
\text { responsáveis pela avaliação. }\end{array}$ & 1 & 1 & 0 & 1 \\
\hline & 6 & 10 & 4 & 7 \\
\hline
\end{tabular}

4- Existência de quadro Mostra se existe uma institucional específico instituição responsável pelo para avaliação processo de avaliação.

A análise confirma a heterogeneidade no nível e forma de institucionalização dos países do MERCOSUL. No caso do Brasil, líder do bloco, o nível de institucionalização é alto (10). Talvez o país precise dar um passo adiante na construção de um organismo centralizado e com autonomia técnica e política para comandar uma política nacional de avaliação. Já a Argentina (6) e Uruguai (7) mostram um nível de institucionalização médio, ambos com projetos em andamento para criar uma agencia nacional de avaliação e monitoramento de políticas públicas, sendo possível que nos próximos ciclos políticos estes dois países avancem para o nível alto. Por último, no caso do Paraguai (4), a institucionalidade se mostrou baixa, pois o país precisa de um quadro jurídico que seja mais compatível com a emergência de uma política nacional de avaliação. Em síntese, só o Brasil apresentou elementos suficientes que justificam a existência de um sistema 
de nacional de avaliação consolidado.

\section{CONCLUSÕES}

A pesquisa buscou aferir o grau de institucionalização alcançado pelos países membros do MERCOSUL (Argentina, Brasil, Paraguai e Uruguai) nos seus sistemas de avaliação e monitoramento de políticas públicas. Para tal, utilizaram-se informações produzidas pelos próprios países, incluindo seu próprio arcabouço jurídico, e outros dados de fontes secundarias como organizações internacionais e ONGs. Os principais resultados da pesquisa mostram que:

(i) - A estrutura institucional vigente nos sistemas de AMPP nos países do MERCOSUL é heterogênea, e o caso brasileiro é o único que mostra elementos suficientes para justificar a existência de uma institucionalidade de AMPP com alto grau de consolidação. Contudo, no caso da Argentina e Uruguai (institucionalidade de AMPP em nível médio de desenvolvimento), existem iniciativas para a criação de agências nacionais dedicadas à avaliação e monitoramento de políticas, o que poderá impactar positivamente ambos sistemas nacionais. Já no caso paraguaio, a análise mostra que a institucionalidade é baixa e ainda se encontra em estágios iniciais.

(ii) - Os quatro países analisados coincidem na ausência de uma política nacional ampla e na falta de um organismo/instituição nacional/federal que coordene o processo de harmonização e unificação do sistema nacional de AMPP. Da mesma forma, os países coincidem na presença de avaliações dispersas e regidas por leis secundárias ou diretrizes operativas criadas por instituições específicas e descentralizadas.

(iii) - Um dos principais desafios para melhorar a institucionalização é a constituição da autonomia técnica e política de um órgão especializado para coordenar todo o sistema nacional de AMPP. Neste sentido, faz-se necessária a atuação estatal para promover e incentivar a criação de uma cultura de avaliação, onde toda a informação e resultados produzidos sejam, de fato, valoradas e utilizadas pela gestão pública.

No âmbito do MERCOSUL, resulta necessária a promoção de mecanismos de fortalecimento institucional dos sistemas nacionais de AMPP mediante a cooperação técnica entre os países membros. Isso pode representar uma pedra angular na implementação e aprimoramento de políticas públicas regionais, como as já existentes para o combate ao trabalho infantil. Por último, se recomenda a criação de novas pesquisas abordando a questão institucional de AMPP a níveis estaduais e municipais, e também com abordagens normativas que contribuam com o desenho e aprimoramento das formas institucionais existentes.

\section{REFERÊNCIAS}

AQUILINO, N.. Hacia una política nacional de evaluación. Buenos Aires: Centro de Implementación de Políticas Públicas para la Equidad y el Crecimiento (CIPPEC), 2015.

AQUILINO, N.; RUBIO, J.; BALLESCÁ, M.; POTENZA, F.. ¿Todos los caminos conducen a Roma? Análisis comparado de la institucionalización de la evaluación. Buenos Aires: Centro de Implementación de Políticas Públicas para la Equidad y el Crecimiento (CIPPEC), 2017.
ARGENTINA. Constitución de la nación argentina. Imprenta Nacional, 1995.

ARGENTINA. Ley 25.152 de administración de los recursos publicos. Información Legislativa y Documental. Buenos Aires, 1999.

ARGENTINA. Resolución JGM 416/2013. Creación del 
Programa de Evaluación de Políticas Públicas. Información Legislativa y Documental. Buenos Aires, 2013.

BID. Banco Interamericano de Desarrollo. La Gestión para Resultados en el Desarrollo. Washington: BID, 2011.

BID. Banco Interamericano de Desarrollo. Los sistemas de monitoreo y evaluación: hacia la mejora continua de la planificación estratégica y la gestión pública. Lima: BID, 2016.

BRASIL. Constituição (1988). Constituição da República Federativa do Brasil. Brasília: DOU, 1988.

BRASIL. Decreto n. 9.588, de 27 de novembro de 2018. Institui o Comitê de Monitoramento e Avaliação dos Subsídios da União. Brasília: DOU, 2018.

BRASIL. Decreto n. 9.834, de 12 de junho de 2019. Institui o Conselho de Monitoramento e Avaliação de Políticas Públicas. Brasília: DOU, 2019a.

BRASIL. Lei n. 13.971, de 27 de dezembro de 2019. Institui o Plano Plurianual da União para o período de 2020 a 2023. Brasília: 2019b.

CARRASCO, G.. Los sistemas de evaluación de las políticas públicas en los países de la Alianza del Pacifico (Colombia, Chile, Perú y México). Edähi Boletín Científico de Ciencias Sociales y Humanidades del Icshu, Pachuca, v.4, n.7, p.1-10, 2015.

CASTRO, V.. Evaluación de políticas públicas: contexto, tendencias y desafíos para la realidad argentina. RevIISERevista de Ciencias Sociales y Humanas, v.6, n.6, p.19-34, 2015.

CRUMPTON, C. D.; MEDEIROS, J. J.; FERREIRA, V. R. S.; SOUSA, M. M.; NAJBERG, E.. Avaliação de políticas públicas no Brasil e nos Estados Unidos: análise da pesquisa nos últimos 10 anos. Rev. Adm. Pública, Rio de Janeiro, v.50, n.6, p.981-1001, 2016.

CUNILL, N.; OSPINA, S.. Fortalecimiento de los sistemas de monitoreo y evaluación (M\&E) en América Latina. Caracas: Banco Mundial/CLAD, 2008.

FEINSTEIN, O.. Sobre el desarrollo de los sistemas de evaluación en América Latina y el Caribe. Revista del CLAD Reforma y Democracia, n.62, p.193-210, 2015.

FITZPATRICK, J.; SANDERS, J.; WORTHEN, B.. Program evaluation: alternative approaches and practical guidelines. 4 ed. New Jersey: Pearson Education, 2011.

FONT, L.. Pequeños pasos, grandes movimientos: Panorama del sistema nacional de monitoreo y evaluación de políticas públicas en Uruguay. In: PANORAMA DE LOS SISTEMAS NACIONALES DE MONITOREO Y EVALUACIÓN EN AMÉRICA LATINA. Anales. CIDE, 2015.

FREIGEDO, M.; MILANESI, A.; RAK, Á.. Dimensiones políticoinstitucionales del desarrollo de sistemas de evaluación. Un análisis a partir del caso uruguayo. Política y cultura, n.47, p.117-141, 2017.

GEVA-MAY, I.; PAL, L. A.. Good fences make good neighbours: policy evaluation and policy analysis-exploring the differences. Evaluation, v.5, n.3, p.259-277, 1999.

GUERSANI, I.. El sistema nacional de evaluación de políticas públicas como recurso institucional transversal para la mejora de las políticas públicas. Disertación (Maestría en Políticas Públicas y Sociales) - Universitat Pompeu Fabra, Barcelona, 2019

HAEFNER, C.; GUTIÉRREZ, M.. Evaluación de políticas públicas y efectividad de la inversión social. Estudio metaevaluativo sobre desempeño de programas sociales. Tzhoecoen, v.11, n.2, p.10-22, 2019.

LAFUENTE, M.. La experiencia del sistema nacional de evaluación del proceso educativo, SNEPE, en Paraguay: aprendizajes y desafíos. RIEE. Revista Iberoamericana de Evaluación Educativa, v.2, n.1, 2009.

LÁZARO, B.. Comparative study on the institutionalisation of evaluation in Europe and Latin America. Programme for Social Cohesion in Latin America, 2015.

MACKAY, K.. Institucionalización de los sistemas de seguimiento y evaluación para mejorar la gestión del sector público. Washington: IEG, 2006.

MASI, F.; GONZÁLEZ, I.; SERVÍN, B.. Investigar en Paraguay: EI desafío en Ciencias Sociales. Estudios Paraguayos, v.34, n.1, p.79-113, 2016.

MÁTTAR, J.; CUERVO, L.. Planificación para el desarrollo en América Latina y el Caribe: enfoques, experiencias y perspectivas. CEPAL, 2017.

NEIROTTI, N.. Evaluación y toma de decisiones: diálogo entre políticos y académicos para fortalecer la democracia. Buenos Aires: Ediciones de la UNLa, 2020.

NOGUEIRA, R. A.; CAVALCANTE, P. L.. Avaliação e monitoramento: duas experiências de institucionalização na gestão das políticas públicas no Poder Executivo Federal brasileiro. In: CONGRESO INTERNACIONAL DEL CLAD SOBRE LA REFORMA DEL ESTADO Y DE LA ADMINISTRACIÓN PÚBLICA, 19. Anais. 2009

OCDE. Glossary of key terms in evaluation and resultsbased management. Paris: OECD Publications, 2002.

PARAGUAY. Decreto N. $\mathbf{4 . 0 7 0}$ del $\mathbf{1 0}$ de noviembre de 2004. Por el cual se reorganiza la Secretaría Técnica de Planificación del Desarrollo Económico y Social, dependiente de la Presidencia de la República. Asunción, 2004.

PARAGUAY. Ley N. 6258/2019. Que aprueba el Presupuesto General de la Nación para el Ejercicio Fiscal 2019. Asunción, 2004.

PARAGUAY. Ley N. 841 del 14 de septiembre de 1962. Por el que se crea la Secretaría Técnica de Planificación del Desarrollo Económico y Social. Asunción, 1962.

QUEIROZ, L.; CAPELARI M.. Condições para avaliação de resultados em políticas públicas: um debate sobre o papel da institucionalidade. Revista de Administração Pública, v.54, n.3, p.433-447, 2020. 
ROSENSTEIN, B.. Mapping the status of national evaluation policies. 2 ed. 2013. DOI:

http://doi.org/10.13140/RG.2.1.2008.0809

TYLER, R. W.. Basic principles of curriculum and instruction. University of Chicago press, 2013.

URUGUAY. Ley N. 17866 de 21 de marzo de 2005. Se crea el Ministerio de Desarrollo Social. Montevideo, 2012.

URUGUAY. Ley N. 18996 de 7 de noviembre de 2012.

Apruébase la Rendición de Cuentas y Balance de Ejecución Presupuestal. Montevideo, 2012.
YARAHUÁN, G.; TRUJILLO, C.. Panorama de los sistemas nacionales de monitoreo y evaluación en América Latina. CIDE, 2015.

ZAMORANO, M.. La evolución de las políticas culturales del Paraguay: hegemonías y transformaciones. Cuadernos de Observación en Gestión y Políticas Culturales, v.1, p.8-23, 2012.

ZAPICO, E.. Evaluación y gobernanza en la Unión Europea. Gestión y análisis de políticas públicas, n.4, p.9-19, 2010. DOI: https://doi.org/10.24965/gapp.v0i4.436

A CBPC - Companhia Brasileira de Produção Científica (CNPJ: 11.221.422/0001-03) detém os direitos materiais desta publicação. Os direitos referem-se à publicação do trabalho em qualquer parte do mundo, incluindo os direitos às renovações, expansões e disseminações da contribuição, bem como outros direitos subsidiários. Todos os trabalhos publicados eletronicamente poderão posteriormente ser publicados em coletâneas impressas sob coordenação da Sustenere Publishing, da Companhia Brasileira de Produção Científica e seus parceiros autorizados. Os (as) autores (as) preservam os direitos autorais, mas não têm permissão para a publicação da contribuição em outro meio, impresso ou digital, em português ou em tradução. 